\title{
Prebiotics can change immunomodulatory properties of probiotics
}

\author{
PAVLINA SREDKOVA, TSVETELINA BATSALOVA, DZHEMAL MOTEN, BALIK DZHAMBAZOV
}

Plovdiv University, Bulgaria

\begin{abstract}
Beneficial effects of probiotics and prebiotics are mainly related to modulation of compositions and activities of gut microbiota as well as manipulation of immunological reactivity in autoimmune diseases. In the present study, we examined whether metabolic products from different strains of Lactobacillus brevis cultured with different prebiotics have similar immunomodulating properties on immune cells under normal and inflammatory conditions, using mouse model of collagen-induced arthritis (CIA). Two strains of Lactobacillus brevis (3448 and 8429) were cultured with four different prebiotics, such as xylooligosaccharides, inulin, pectin, and chitosan. Sterile supernatants containing different metabolic products have been used for direct treatment of cell cultures prepared from CII-immunized mice and non-immunized (control mice). Our results showed that metabolic products from XOS decreased levels of IFN- $\gamma, I L-6, I L-17$, and TNF- $\alpha$ in both cultures from immunized and non-immunized mice. In contrast, metabolic products from inulin, pectin, and chitosan increased concentrations of these cytokines with highest values for pectin. Neither of investigated prebiotics influenced the secretion of IL-10. In addition, we found changes in the percentage of macrophages, which were different for the tested prebiotics. Also, metabolic products from pectin and chitosan caused loss of T-cells $(C D 3+)$ and increased percentages of $\mathrm{CD}^{+} \mathrm{CD}_{25} 5^{+}$regulatory $\mathrm{T}$ cells and $\mathrm{CD} 8^{+} \mathrm{CD} 279^{+}$anergic $\mathrm{T}$ cells. Hence, our data indicate that immunomodulating properties of probiotics are strain-specific and prebiotic-dependent.
\end{abstract}

Key words: cytokines, probiotics, immunomodulation, inflammation, prebiotics, Lactobacillus brevis.

(Cent Eur J Immunol 2020; 45 (3): 248-255)

\section{Introduction}

Gut microbiota has a major impact on gastrointestinal metabolism and nutrient absorption, and thereby plays a critical role in maintaining good health in humans. During the last years, different probiotics and prebiotics have been suggested as an effective approach for health improvement by using their capacity to modulate the immune system. The immune response to microbiota is realized mainly via Toll-like receptors (TLRs) expressed on macrophages, neutrophils, epithelial cells, dendritic cells, and $\mathrm{T}$ - and B-cells $[1,2]$. It has been shown that probiotics may induce production of TNF- $\alpha$, IL-1b, IL-6, IL-10, IL-12, and IL-23, and also stimulate other non-specific signal pathways of the immune system [3, 4]. Lactobacillus and Bifidobacterium are the most used genera for isolation of probiotic strains with desired beneficial effects [5].

Composition of gut microbiota, appropriate balance between commensal microorganisms and pathogens as well as microbial-produced signals play essential roles in the immune development, homeostasis, and tolerance [6]. Disruption of this balance may cause autoimmune disorders associated with chronic inflammation, such as inflammatory bowel disease (IBD) or rheumatoid arthritis (RA).
As an autoimmune chronic disease, RA is characterized by joint inflammation, significant pain, stiffness, progressive joint destruction, and functional disability. Few data are available on the effects of probiotic supplementation in patients with RA. Oral intake of Lactobacillus casei 01 alone or a probiotic mix of L. acidophilus, L. casei, and Bifidobacterium bifidum for 8 weeks resulted in improved disease activity score of 28 joints (DAS-28), reduced production of proinflammatory cytokines (IL-1 $\beta$, IL-6, IL-12, IL-17, IFN- $\gamma$, and TNF- $\alpha$ ), decreased serum levels of insulin and high-sensitivity C-reactive protein (hs-CRP), and increased concentrations of IL-10 and TGF- $\beta$ in patients with RA compared with a placebo group of patients $[1,2,7]$. Also, in several studies performed with animal models for RA, oral administration of probiotics presented decreased levels of proinflammatory cytokines, lower incidence, and reduced clinical arthritis scores [8-12].

In addition to IBD and RA, it was proposed that various other diseases and dysfunctions are associated with an imbalance or dysregulation of the microbiota $[13,14]$. Hence, the restoration of this balance and treatment of associated disorders by using probiotics, prebiotics, or their combinations (synbiotics) is a new challenge for clinical 
studies. Since probiotics affect the immune responses in a strain-specific manner $[15,16]$, most of the researchers are trying to find bacterial strains with specific beneficial properties and to enhance these properties by using selective substrates (prebiotics), which facilitate the growth of probiotic bacteria. Although the oligosaccharides are the most common used prebiotics, the list of new prebiotic candidates is steadily growing. Different non-digestible carbohydrates used as energy sources can modify the gut microbiota in various ways promoting different bacterial species, and these modifications were found at a level of individual strains and species rather than at a phylum or family level [17]. Therefore, the beneficial effects of probiotics on the human health are strain- and prebiotic-dependent.

In the present study, we investigated the immunomodulating properties of metabolic products from two strains of Lactobacillus brevis (3448 and 8429) cultured with four different prebiotics (xylooligosaccharides, inulin, pectin, and chitosan). A cytokine secretion and profile of immune cells in inflammatory conditions using a collagen-induced arthritis (CIA) model, an animal model for RA, were also examined.

\section{Material and methods}

\section{Bacterial strains (probiotics) and supplements (prebiotics) tested}

Lactobacillus brevis NBIMCC 3448 and L. brevis NBIMCC 8429 were purchased from the National Bank for Industrial Microorganisms and Cell Cultures (NBIMCC), Sofia, Bulgaria. The bacterial cultures were obtained by using $0.2 \%$ inoculum from stocks stored at $-80^{\circ} \mathrm{C}$ in de Man, Rogosa and Sharpe (MRS) medium containing 20\% glycerol. After thawing, Lactobacillus strains were grown in MRS broth (Merck, Germany) at $37^{\circ} \mathrm{C}$ for $18-24$ hours. MRS cultures were then subcultured in fresh Dulbecco's modified Eagle's (DMEM) medium (Gibco, Invitrogen, USA) without antibiotics and fetal calf serum (FCS). The MRS cultures were centrifuged at $4000 \mathrm{rpm} / \mathrm{min}, 4^{\circ} \mathrm{C}$ for 15 minutes and, after removal of the MRS supernatant, the cells were resuspended in DMEM and divided in separate vessels before prebiotics addition. Prebiotic solutions were added to the bacterial cultures to a final concentration of $15 \mathrm{mg} / \mathrm{ml}$. Control samples included prebiotic-free DMEM. The cultures were grown for 18 hours at $37^{\circ} \mathrm{C}$. At the end of incubation period, the cultures were centrifuged for 15 minutes at $4000 \mathrm{rpm} / \mathrm{min}, 4^{\circ} \mathrm{C}$, and cell-free supernatants were sterilized using Minisart ${ }^{\circledR} \mathrm{NY} 250.2 \mu \mathrm{m}$ with polyamide syringe filters (Sartorius Lab Instruments, Goettingen, Germany). The sterile culture supernatants were stored at $-20^{\circ} \mathrm{C}$ prior in vitro experiments.

Four different commercially available prebiotic substances were tested, including xylo-oligosaccharide (XOS;
Shandong Longlive Bio-Technology, Shandong, China), inulin (Haya Labs, Washington DC, USA), apple pectin (Sigma-Aldrich, Saint Louis, MO, USA), and chitosan 1000 (Equilibra, Torino, Italy). XOS, inulin, and pectin were dissolved in sterile DMEM without antibiotics and FCS, and stored at $4^{\circ} \mathrm{C}$ until use. The chitosan 1000/chromium chloride powder was dissolved in sterile DMEM containing $0.1 \mathrm{M}$ acetic acid to prepare a stock solution of $50 \mathrm{mg} / \mathrm{mL}$.

\section{Animals and induction of arthritis}

DBA/1 mice were originally obtained from Jackson Laboratories (Bar Harbor, ME, USA). A total of 8 male DBA/1 mice ( 8 weeks old, 19-22 g) were used in this study. Mice were kept in a climate-controlled environment, with $12 \mathrm{~h}$ light/dark cycles in polystyrene cages containing wood shavings. They were fed standard rodent chow and water ad libitum under standardized and pathogen-free conditions.

For induction of collagen-induced arthritis (CIA), four mice were injected with $100 \mu \mathrm{g}$ of bovine CII (Sigma-Aldrich, Saint Louis, MO, USA), emulsified 1:1 in CFA (Difco Laboratories, Detroit, MI, USA) at the base of tail, with a total volume of $100 \mu$. The other four mice (control group) were injected with $100 \mu \mathrm{l}$ sterile DPBS (Gibco, Life Technologies, Grand Island, NY, USA). Fourteen days later, mice were sacrificed, and draining lymph nodes and spleens were taken to analyze in vitro cytokine production and cellular immune phenotype. Lymph node cells (LNC) were isolated and passed through a $40 \mu \mathrm{m}$ cell strainer $\left(\mathrm{FALCON}^{\circledR}{ }^{\circledR}\right.$, Becton Dickinson, Le Pont De Claix, France). Single cell suspensions were washed twice with DPBS (Gibco, Life Technologies, Grand Island, NY, USA). Spleens were removed, homogenized, and red blood cells were lysed with $0.84 \%$ ammonium chloride solution (pH 7.4). After passing the cells through a $40 \mu \mathrm{m}$ cell strainer, cells were collected by centrifugation and washed twice with serum-free DMEM. Isolated LNC and splenocytes from the four CII-immunized mice were pooled. The same was done with the cells from non-immunized animals (control mice). Then, the prepared two groups of cells (primed and control) were used in the in vitro assays.

All animals-related procedures were performed according to the European Community regulations for animal experiments and were approved by the local Ethical Committee for Animal Experiments (approval number: 94/11.03.2014).

\section{In vitro activation assay}

The prepared cells were adjusted to $1 \times 10^{6}$ cells $/ \mathrm{mL}$ and re-stimulated with $50 \mu \mathrm{g} / \mathrm{mL}$ bovine CII in DMEM supplemented with $10 \%$ heat-inactivated FCS (Gibco, Life Technologies, Grand Island, NY, USA), 100 U/mL penicillin, and $100 \mu \mathrm{g} / \mathrm{ml}$ streptomycin (Sigma-Aldrich, Ger- 
many). The cells were cultured in 96-well plates (Thermo Fisher Scientific, Nunclon ${ }^{\mathrm{TM}}$ Delta Surface, Roskilde, Denmark) at $37^{\circ} \mathrm{C}$ in a $5 \% \mathrm{CO}_{2}$-humidified incubator for $48 \mathrm{~h}$ in the presence of $20 \%$ metabolic products (supernatants from the probiotic strains cultured with different prebiotic supplements). The control samples were supplemented with DMEM. All samples were plated in triplicates. At the end of incubation period, supernatants from each sample well were collected for measurement of cytokines and frozen at $-20^{\circ} \mathrm{C}$ until further use. The cells were analyzed immediately by flow cytometry.

\section{Enzyme-linked immunosorbent assay (ELISA) for cytokine detection}

The levels of IFN- $\gamma$, IL-6, IL-17A, TNF- $\alpha$, and IL-10 cytokines in the supernatants collected after in vitro stimulation were measured using commercially available specific ELISA kits from R\&D Systems Inc. (Minneapolis, MN, USA) according to the manufacturer's recommendations. The absorbance was measured at $450 \mathrm{~nm}$ in a microplate reader $\left(\mathrm{ELx} 800^{\mathrm{TM}}\right.$, BioTek Instruments, Winooski, VT, USA) and cytokine concentrations were calculated according to the standard curves from values of recombinant cytokines.

\section{Flow cytometry (FACS) analysis}

At the end of in vitro stimulation, the cells were stained by using a 10-color panel from fluorochrome-labeled antibodies directed to different murine cell surface markers, including CD3, CD4, CD8, CD11b, CD19, CD25, CD45, CD279(PD-1), MHCII, and LIVE/DEAD (BD Biosciences, San Jose, CA, USA). The flow-cytometric analysis was performed according to standard settings on an LSR II flow cytometer (BD Biosciences, San Jose, CA, USA). Debris and dead cells were excluded from the evaluation by common gating procedures. Flow cytometry data were analyzed by FlowJo software (FlowJo LLC, USA).

\section{Statistical analysis}

Results are expressed as mean values \pm standard deviation (SD). To compare non-parametric data for statistical significance, the Mann-Whitney U-test or Kruskal-Wallis test were applied using StatView program (SAS Institute). Values of $\mathrm{p}<0.05$ were considered significant. All results were compared to those from the controls.

\section{Results}

This study was designed to evaluate whether the metabolic products from two strains of Lactobacillus brevis (3448 and 8429) cultured with four different prebiotics as energy sources provide similar immunomodulatory effects under normal and inflammatory conditions. As shown in Figure 1A, the production of IFN- $\gamma$ significantly decreased in both groups (control and immunized mice), when the murine cells were co-cultured with metabolic products received from L. brevis 3448 and xylooligosaccharides (XOS). Also, similar results were obtained for the other probiotic strain (L. brevis 8429), when it was grown with XOS (Fig. 1B). In contrast, metabolic products obtained from $L$. brevis 3448 grown with other prebiotics (inulin, pectin, and chitosan) showed elevated secretion of IFN- $\gamma$ with highest values for pectin (Fig. 1A). For the other strain (L. brevis 8429), an increased production of IFN- $\gamma$ with statistical significance was observed only for the pectin in both cell cultures, from non-immunized and CII-immunized mice (Fig. 1B). Data showed that metabolic products from the 8429-strain cultivated with the other prebiotics (inulin and chitosan) did not significantly change the production of IFN- $\gamma$ compared to the controls (Fig. 1B).

Metabolic products from both bacterial strains (3448 and 8429) and XOS also inhibited IL-6 secretion compared with the control group in murine cultures from immunized and non-immunized mice (Fig. 1C and 1D). This decrease was most prominent in L. brevis 3448-strain and in the group of CII-immunized mice (Fig. 1C). Increased levels of IL- 6 were found in all murine cell cultures after the treatment with metabolic products from pectin and chitosan, with higher values for the group of immunized and CII-re-stimulated mice (Fig. 1C and 1D). Slightly, but statistically significant increased production of IL-6 was observed only in the group of immunized mice when the cells were co-cultured with metabolic products from L. brevis 3448-strain and inulin (Fig. 1C). With few exceptions, it seems that the combination of L. brevis and inulin as a prebiotic does not lead to immunomodulatory effects on mammalian cell cultures.

ELISA test results showed lower levels of IL-17 compared to the controls in the group of non-immunized mice, when the immune cells were treated with metabolic products from L. brevis 3448-strain grown on either XOS or inulin (Figure 1E). Increased concentrations of IL-17 were detected in this group as well as in the group of immunized mice, when the cells were co-cultured with products from L. brevis 3448 grown on pectin and chitosan (Fig. 1E). Regarding L. brevis 8429-strain, we detected lower concentration of IL-17 after growing with XOS and higher concentration of IL-17 after growing with pectin. These changed were observed only in the group of immunized mice (Fig. 1F).

All supernatants derived from the culturing of L. brevis 3448 with four different prebiotics (XOS, inulin, pectin, and chitosan) resulted in a stimulation of TNF- $\alpha$ secretion in the group of cell cultures from the control (non-immunized) mice (Fig. 2A). In the group of immunized mice, metabolic products after growing with XOS reduced the production of TNF- $\alpha$ compared to the control, while those from the growth with pectin and chitosan, increased the concentration of TNF- $\alpha$ (Fig. 2A). Again, inulin had no 


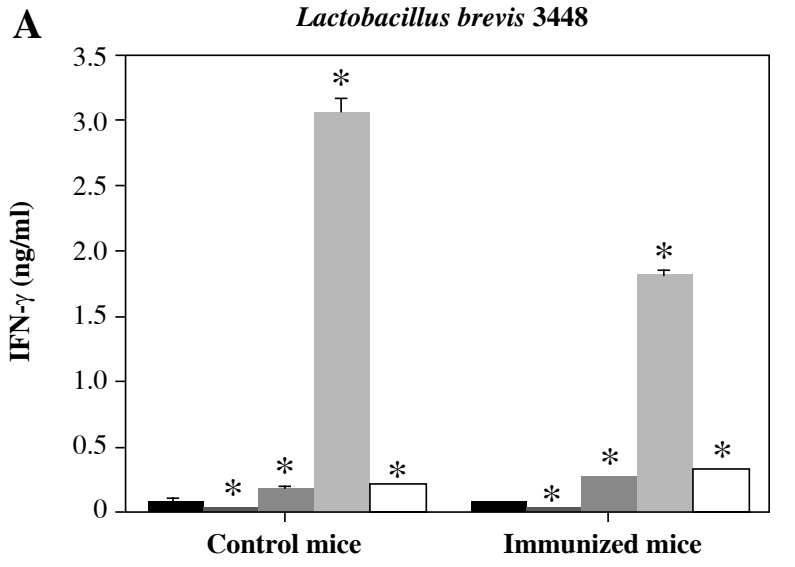

Medium $\square$ XOS $\square$ Inulin $\square$ Pectin $\square$ Chitosan

C

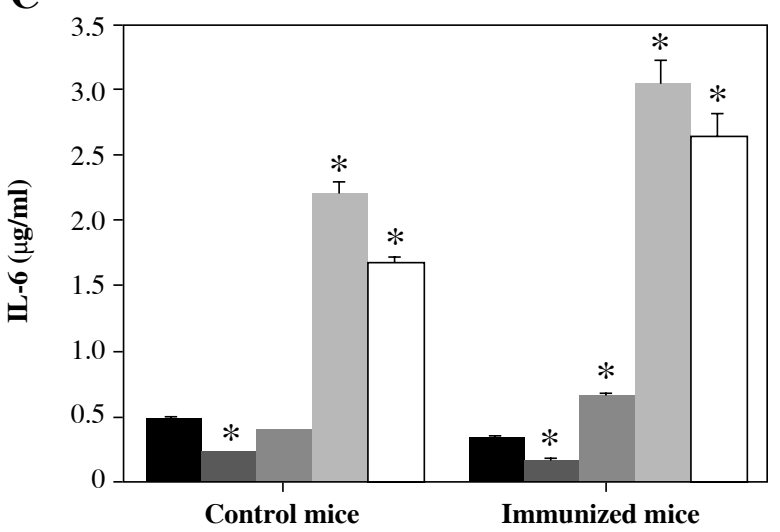

Medium $\square$ XOS $\square$ Inulin $\square$ Pectin $\square$ Chitosan

E

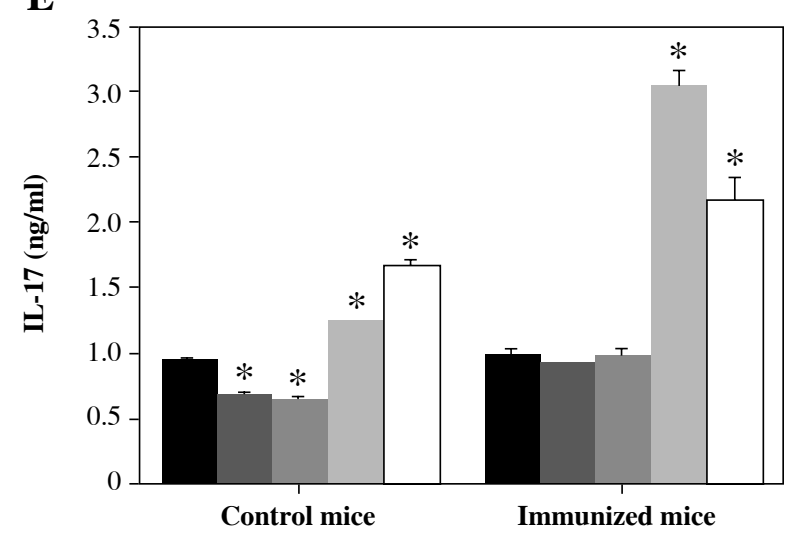

Medium XOS Inulin Pectin $\square$ Chitosan

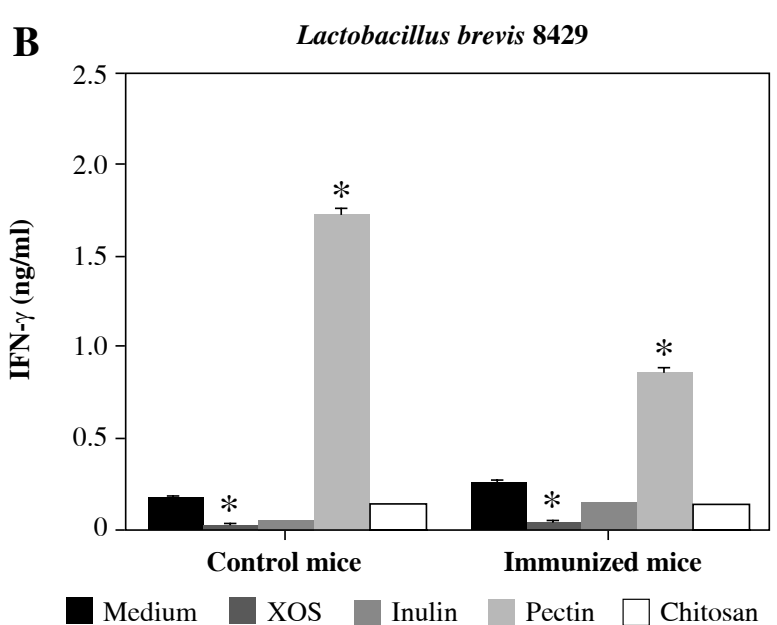

D

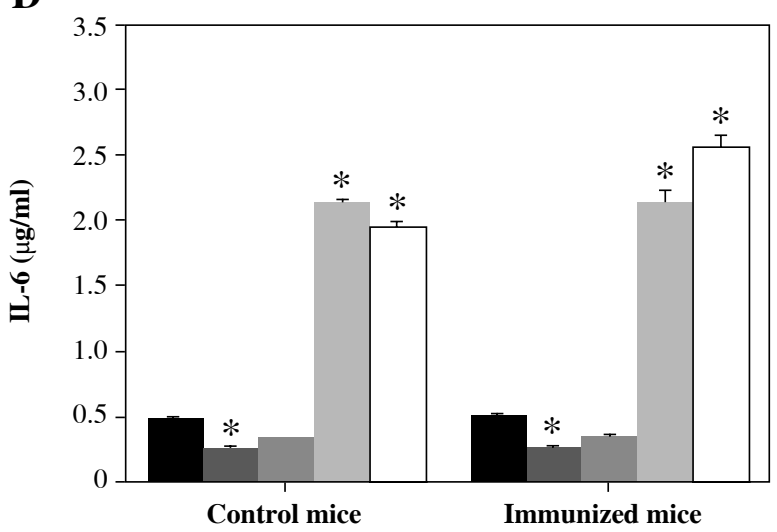

Medium $\square$ XOS $\square$ Inulin $\square$ Pectin $\square$ Chitosan

F

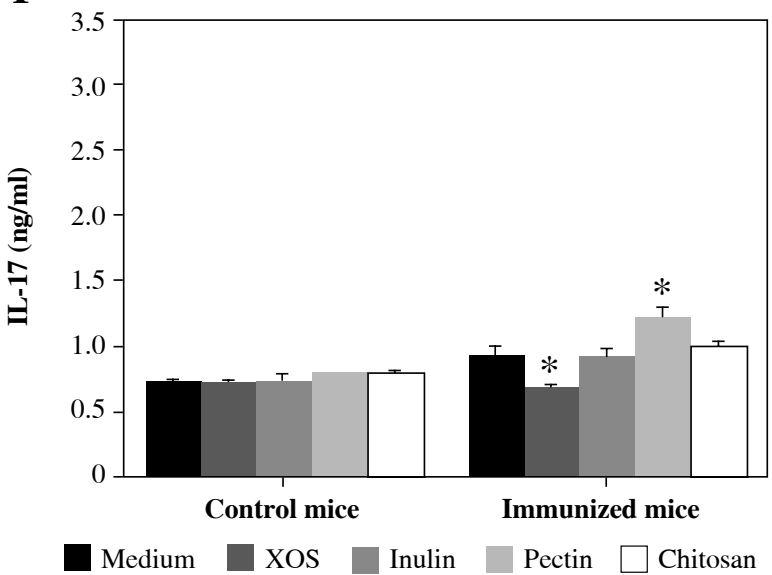

Fig. 1. Effects of the metabolic products from two strains of Lactobacillus brevis grown with different prebiotics (xylooligosaccharides, inulin, pectin, and chitosan) on the cytokine production of IFN- $\gamma$, IL-6, and IL-17 in murine cultures prepared from lymph nodes and splenocytes from non-immunized (control) mice and CII-immunized mice after $48 \mathrm{~h}$ of exposure. As negative controls, cell cultures without stimulation with metabolites from prebiotics (only medium) were used. Results are expressed as mean \pm SE of triplicates, with $* p<0.05$ 
A

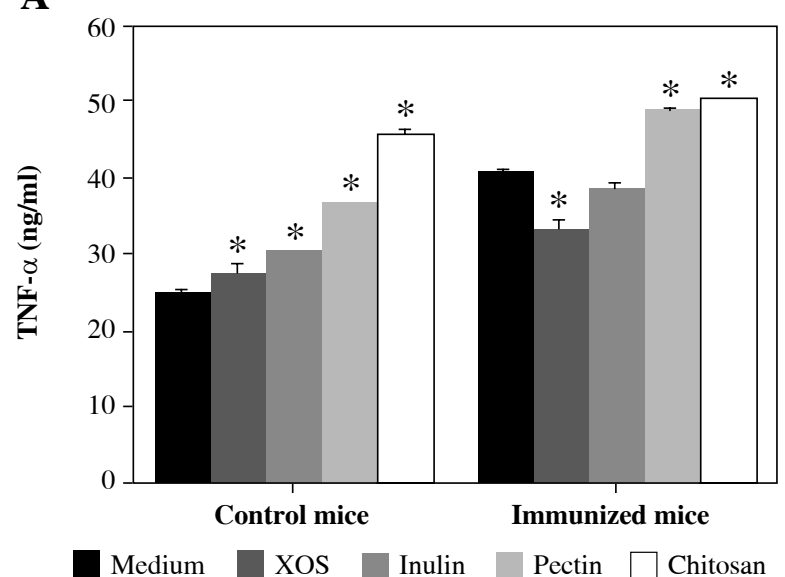

B

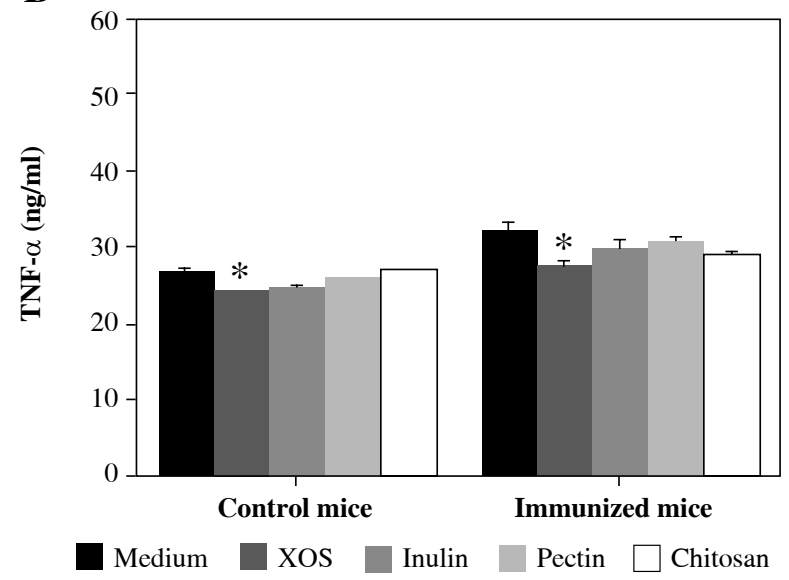

C

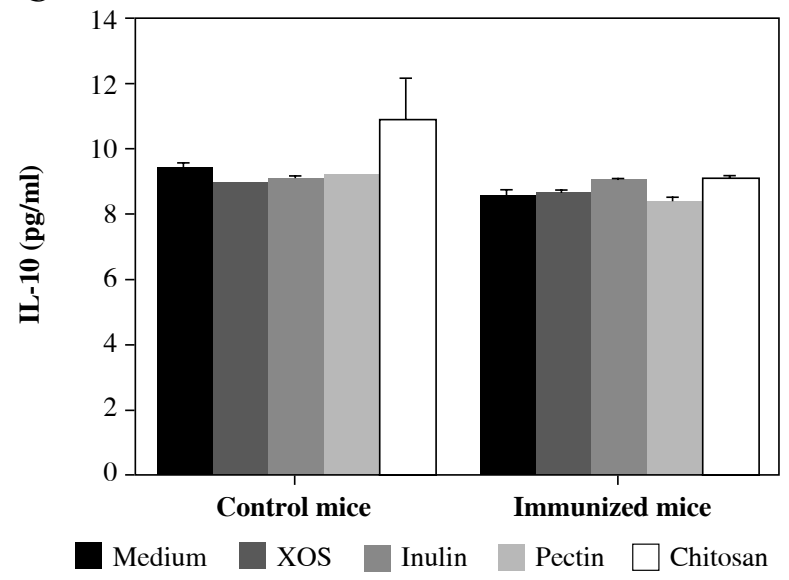

D

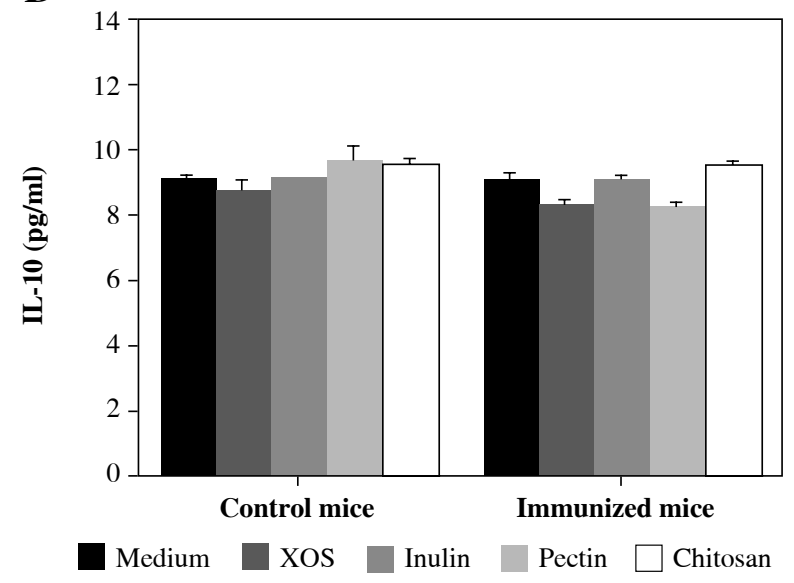

Fig. 2. Influence of the metabolic products from examined probiotic strains and prebiotics on the secretion of TNF- $\alpha$ and IL-10 in murine cultures prepared from lymph nodes and splenocytes from non-immunized (control) mice and CII-immunized mice after $48 \mathrm{~h}$ of exposure. As negative controls, cell cultures without supplementations (only medium) were used. Results are expressed as mean \pm SE of triplicates, with $* p<0.05$

effect on the cytokine production from the immune cells under inflammatory conditions. When we tested the supernatants from $L$. brevis 8429-strain grown with investigated prebiotics, only XOS had a significant effect on the production of TNF- $\alpha$, decreasing its concentration in both cultures, from the control and immunized mice (Fig. 2B).

Surprisingly, no cell from the supernatants derived from the two L. brevis strains grown in the presence of the four prebiotics had an effect on the production of IL-10 neither in the control nor in the immunized mice groups (Fig. 2C and 2D).

We also performed FACS analysis to determine whether the investigated metabolic products can induce changes in the main immune cell subsets. FACS analysis showed that expression of $\mathrm{CD}^{4} 5^{+}$as a whole, decreased in all samples from the immunized mice compared to those from the control (non-immunized) mice (Table 1), even for the non-treated cells (medium only). As expected, this tendency remains for the next marker $\left(\mathrm{CD} 19^{+}\right)$indicating that the decrease is related to reduction of B-cells due to their differentiation into plasma cells after immunization/ activation, but not to the tested metabolic products. When we analyzed the T-cell population $\left(\mathrm{CD}^{+}\right)$, we found a reduction of T-cells after the treatment with metabolic products from pectin and chitosan, and this effect was stronger in cultures from the control mice (Table $1, \mathrm{CD}^{+}$, in bold). Consequently, this is the reason for an additional decrease of $\mathrm{CD} 45^{+}$cells after the treatment with pectin or chitosan (Table 1, CD45 ${ }^{+}$, in bold), which means a loss of T-cells. Probably, in the cultures from CII-immunized mice, this loss is partially compensated by the proliferation of CII-specific T cells after CII re-stimulation. Significant 
Table 1. Analysis of the immune cell subsets after in vitro re-stimulation of the murine cell cultures with CII and co-culture with metabolic products derived from two L. brevis strains and four prebiotics (XOS, inulin, pectin, and chitosan). The cells were stained using a 10-color panel of indicated surface markers and analyzed by FACS. Percentages of specific populations from the total number of cells are shown

\begin{tabular}{|c|c|c|c|c|c|c|c|c|c|c|}
\hline Prebiotics & $\mathrm{CD}^{+}{ }^{+}$ & $\mathrm{CD}^{+}{ }^{+}$ & $\mathrm{CD3}^{+}$ & $\mathrm{CD4}^{+}$ & $\mathrm{CD8}^{+}$ & $\begin{array}{c}\mathrm{CD4}^{+} \\
\mathrm{CD25}^{+}\end{array}$ & $\begin{array}{c}\mathrm{CD4}^{+} \\
\mathrm{CD279}^{+}\end{array}$ & $\begin{array}{c}\text { CD8 }^{+} \\
\text {CD279 }^{+}\end{array}$ & $\begin{array}{c}\text { CD11b }^{+} \\
\text {MHCII }^{+}\end{array}$ & Group \\
\hline \multicolumn{11}{|c|}{ L. brevis 3448} \\
\hline Medium & 89.1 & 53.3 & 27.2 & 16.2 & 7.28 & 2.59 & 2.70 & 0.58 & 5.34 & \multirow{5}{*}{$\begin{array}{l}\text { Control } \\
\text { mice }\end{array}$} \\
\hline XOS & 88.9 & 52.5 & 27.9 & 16.3 & 7.55 & 2.47 & 2.59 & 0.63 & 5.34 & \\
\hline Inulin & 88.8 & 48.9 & 27.8 & 16.7 & 7.37 & 2.88 & 2.70 & 0.60 & 9.08 & \\
\hline Pectin & 83.2 & 48.7 & 24.8 & 15.7 & 6.78 & 4.35 & 2.67 & 0.93 & 6.10 & \\
\hline Chitosan & 85.4 & 49.6 & 25.0 & 16.9 & 7.27 & 3.55 & 2.47 & 0.94 & 8.44 & \\
\hline Medium & 83.8 & 46.5 & 28.1 & 17.3 & 8.34 & 3.86 & 2.27 & 0.64 & 4.43 & \multirow{5}{*}{$\begin{array}{l}\text { Immunized } \\
\text { mice }\end{array}$} \\
\hline XOS & 83.6 & 45.8 & 28.6 & 17.6 & 8.63 & 3.88 & 2.34 & 0.68 & 4.58 & \\
\hline Inulin & 83.3 & 44.7 & 29.0 & 17.8 & 8.44 & 3.62 & 2.45 & 0.70 & 5.38 & \\
\hline Pectin & 80.8 & 45.4 & 25.9 & 16.5 & 7.96 & 4.14 & 2.62 & 0.80 & 5.33 & \\
\hline Chitosan & 83.0 & 46.4 & 27.1 & 17.8 & 8.74 & 4.16 & 2.84 & 0.85 & 6.97 & \\
\hline \multicolumn{11}{|c|}{ L. brevis 8429} \\
\hline Medium & 88.1 & 48.7 & 26.7 & 16.2 & 7.24 & 2.42 & 2.98 & 0.53 & 5.00 & \multirow{5}{*}{$\begin{array}{c}\text { Control } \\
\text { mice }\end{array}$} \\
\hline XOS & 89.3 & 50.7 & 28.1 & 17.3 & 7.56 & 2.48 & 2.43 & 0.52 & 4.92 & \\
\hline Inulin & 89.3 & 48.1 & 28.1 & 17.4 & 7.25 & 2.94 & 2.68 & 0.50 & 10.5 & \\
\hline Pectin & 83.5 & 50.5 & 25.3 & 15.7 & 6.49 & 5.19 & 2.57 & 1.17 & 3.44 & \\
\hline Chitosan & 87.4 & 49.4 & 24.9 & 16.6 & 7.34 & 3.83 & 2.87 & 0.71 & 11.3 & \\
\hline Medium & 81.8 & 44.7 & 26.8 & 16.8 & 8.38 & 3.85 & 2.45 & 0.57 & 4.68 & \multirow{5}{*}{$\begin{array}{l}\text { Immunized } \\
\text { mice }\end{array}$} \\
\hline XOS & 82.7 & 46.9 & 27.6 & 17.5 & 8.58 & 3.48 & 2.49 & 0.67 & 3.73 & \\
\hline Inulin & 83.0 & 44.3 & 27.4 & 17.1 & 8.65 & 3.47 & 2.48 & 0.59 & 7.09 & \\
\hline Pectin & 81.1 & 46.9 & 27.0 & 16.8 & 8.12 & 4.98 & 2.55 & 0.95 & 2.80 & \\
\hline Chitosan & 83.0 & 44.5 & 27.3 & 18.4 & 8.69 & 3.85 & 2.48 & 0.83 & 8.48 & \\
\hline
\end{tabular}

changes within $\mathrm{CD}^{+}$and $\mathrm{CD} 8^{+} \mathrm{T}$ cell populations were not found (Table 1).

Next, we decided to verify whether the metabolic products from investigated prebiotics can particularly affect the cells with immunoregulatory properties $\left(\mathrm{CD} 4^{+} \mathrm{CD} 25^{+}\right)$. We observed that supernatants from both $L$. brevis strains (3448 and 8429) grown with pectin as well as L. brevis 3448 grown with chitosan lead to enhancement of the recently activated $\mathrm{T}$ cells with regulatory properties (Table $1, \mathrm{CD} 4^{+} \mathrm{CD} 25^{+}$, in bold). In the non-treated cells from the immunized mice (medium), the percentage of $\mathrm{CD} 4^{+} \mathrm{CD} 25^{+}$ cells was also increased compared to the non-treated cells from the control mice (medium), which was due to the induced inflammatory conditions (Table 1). The fact that tested metabolic products caused similar enhancement of $\mathrm{CD} 4{ }^{+} \mathrm{CD} 25^{+}$cells in both cultures from the immunized and control mice, suggests that this effect is not dependent from the inflammation.

To examine the involvement of probiotics/prebiotics in the PD-1 signaling pathway, we analyzed the changes within CD279 (PD-1) expressing T cells. No changes were detected in the $\mathrm{T}$ cell subset $\mathrm{CD} 4^{+} \mathrm{CD} 279^{+}$(Table 1). FACS analysis of $\mathrm{CD} 8^{+} \mathrm{CD} 279^{+} \mathrm{T}$ cells showed slightly increased percentages of these cells in the case of pectin and chitosan in the cultures from both experimental groups, the control and immunized mice (Table $1, \mathrm{CD} 8{ }^{+} \mathrm{CD} 279^{+}$, in bold).

Neutrophils and macrophages are the first cells that migrate towards the site of inflammation or injury. Since neutrophils are short-lived cells, we analyzed whether investigated bacterial metabolites have some effects on the macrophages. The metabolic products from XOS had no effects or slightly decreased the percentage of macro- 
phages (Table 1, CD11 b $\mathrm{MHCII}^{+}$). Metabolites from the other prebiotics (inulin, pectin, and chitosan) increased the number of macrophages in different degrees compared to the controls. An exception was found in the case of strain L. brevis 8429-strain grown in pectin, where in both cultures (from the control and immunized mice), a decreased number of macrophages was observed (Table 1, CD$11 \mathrm{~b}^{+} \mathrm{MHCII}^{+}$, in bold).

\section{Discussion}

The immunomodulatory properties and health benefits of probiotic bacteria are strain-specific. This phenomenon was reviewed in detail by Skivka [18]. In addition, a particularly important factor that defines the immunomodulating potential of probiotics is the used prebiotic and resulted metabolic products. Most in vivo studies with oral administration of probiotics did not provide precise control on the substrates available to the bacteria since many other food components would be always present. Therefore, the immune mechanisms by which probiotic microorganisms exert their beneficial effects are still not completely understood. Hence, the disagreement between the data of different studies could be due to the used probiotic strain, prebiotics, or different experimental conditions, such as target diseases, cells, or antigens.

In the present study, our strategy was to examine direct effects of the metabolic products resulting from culturing of two L. brevis strains with four different prebiotics on the secretion of some cytokines and phenotype of the immune cells under normal and inflammatory conditions. In this way, we could predict the effects of certain probiotic-prebiotic combination in the case of a preventive protocol on healthy individuals or a therapeutic approach of individuals with a particular disease.

Most studies consider the indirect effects of probiotics/prebiotics on the immune system (through changing the gut microbiota) mainly by inhibiting the expression of proinflammatory cytokines and increased levels of anti-inflammatory cytokines, but evidences about their direct effects are still limited [19]. The data presented here clearly show that metabolites from L. brevis and XOS reduce the levels of IFN- $\gamma$, IL-6, IL-17, and TNF- $\alpha$, but the concentration of IL-10 was not affected. This effect was similar in both cases, non-stimulated cells (from the control mice) and stimulated cells (from the immunized mice). Consistent with our results, a decreased serum levels of IFN- $\gamma$ and IL-1 $\beta$ as well as no effect on IL-10 production was also observed in XOS-fed mice compared to control-fed mice [20]. As a possible mechanism, the authors proposed an indirect SCFA-mediated anti-inflammatory effect of XOS [20]. Our results from the direct test of metabolic products from XOS (including SCFA) on the immune cells confirmed that these products decrease the level of proinflammatory cytokines.
Several clinical trials related to the effects of probiotic supplementation on disease activity and inflammatory cytokines in patients with RA [1, 2, 7] and in vivo studies with animal models for RA [9-12] also showed decreased levels of IL-1 $\beta$, IL-2, IL-6, IL-12, IL-17, TNF- $\alpha$, and IFN- $\gamma$ as well as increased concentrations of the anti-inflammatory cytokines IL-10 and TGF- $\beta$. In these studies, probiotic strains from Lactobacillus casei, L. acidophilus, L. delbrueckii subsp. bulgaricus, and Bifidobacterium bifidum (separately or in a combination) grown in MRS medium or MRS + $10 \%$ skimmed milk (for $L$. delbrueckii) without supplementation with prebiotics were orally administered. When we examined the direct effect of metabolic products from $L$. brevis and inulin, pectin, or chitosan, we observed opposite results. In contrast to the referred reports and to our data from XOS, the levels of IFN- $\gamma$, IL-6, IL-17, and TNF- $\alpha$ increased compared to the controls with highest values for the metabolites from pectin. Similar to the results from XOS, the production of IL-10 was not influenced. These data suggest that immunomodulating properties of probiotics are dependent not only on the strain, but also on the substrate where they are grown. This means that one probiotic strain can exert different immunomodulating properties on the immune system depending on the food diet and the available nutrient components for the gut microbiota. Theoretically, applying a wrong combination between the probiotic strain and used prebiotic or fiber diet for a treatment of certain disease may cause adverse, instead of beneficial effects. Additionally, many other defense mechanisms need to be considered when probiotics, prebiotics, or synbiotics are used for a disease treatment.

It has been shown that the indirect immunomodulating activity of probiotics/prebiotics is due to SCFA, which activate G-protein coupled receptors 41 and 43 (GPR41 and GPR43) on the intestinal epithelial cells, resulting in rapid production of different cytokines and chemokines [21]. Probably, these receptors are also involved in the obtained direct immunomodulatory effects of metabolic products (containing SCFA) tested in the present study, since they are expressed by the macrophages/monocytes as well [22]. Our FACS analyses indicated differences in the number of macrophages depending on prebiotics used. Macrophages may produce IFN- $\gamma$, IL-6, TNF- $\alpha$, and IL-12 [9], leading to subsequent activation of $\mathrm{T}$ cells and secretion of other cytokines (e.g., IL-17). We demonstrated that different prebiotics have distinct effects on the cytokine production. These changes in the cytokine balance can change the immune response in different directions under certain conditions. Treatment of cells with metabolic products from L. brevis and pectin or chitosan increased the number of anergic $\mathrm{CD}^{+} \mathrm{CD} 279^{+} \mathrm{T}$ cells and regulatory $\mathrm{CD} 4{ }^{+} \mathrm{CD} 25^{+}$ $\mathrm{T}$ cells, but the metabolites from XOS had no such effect. Since in mice, $\mathrm{CD}^{+}$Tregs are a homogenous population, in which all $\mathrm{CD} 4{ }^{+} \mathrm{CD} 25^{+}$cells are regulatory $\mathrm{T}$ cells, we did not include the intracellular marker FoxP3. 
In conclusion, metabolic products resulting from culturing of different probiotic strains with different prebiotics could have different effects on the immune response. This could be of crucial importance, when probiotics/prebiotics/ synbiotics are used for treatment of autoimmune diseases.

The authors declare no conflict of interest.

\section{References}

1. Vaghef-Mehrabany E, Alipour B, Homayouni-Rad A, et al. (2014): Probiotic supplementation improves inflammatory status in patients with rheumatoid arthritis. Nutrition 30: 430435.

2. Zamani B, Golkar HR, Farshbaf S, et al. (2016): Clinical and metabolic response to probiotic supplementation in patients with rheumatoid arthritis: a randomized, double-blind, placebo-controlled trial. Int J Rheum Dis 19: 869-879.

3. Isolauri E, Salminen E, Salminen S (1998): Lactic acid bacteria and immune modulation. In: Lactic acid bacteria: microbiology and functional aspects (2nd ed.), Salminen S, von Wright A (eds.). Marcel Dekker, New York: 255-268.

4. Evrard B, Coudeyras S, Dosgilbert A, et al. (2011): Dosedependent immunomodulation of human dendritic cells by the probiotic Lactobacillus rhamnosus Lcr35. PLoS One 6: e18735.

5. Conway PL (1996): Selection criteria for probiotic microorganisms. Asia Pac J Clin Nutr 5: 10-14.

6. Peterson CT, Sharma V, Elmen L, Peterson SN (2015): Immune homeostasis, dysbiosis and therapeutic modulation of the gut microbiota. Clin Exp Immunol 179: 363-377.

7. Alipour B, Homayouni-Rad A, Vaghef-Mehrabany E, et al. (2014): Effects of Lactobacillus casei supplementation on disease activity and inflammatory cytokines in rheumatoid arthritis patients: a randomized double-blind clinical trial. Int J Rheum Dis 17: 519-527.

8. Kato I, Endo-Tanaka K, Yokokura T (1998): Suppressive effects of the oral administration of Lactobacillus casei on type II collagen-induced arthritis in DBA/1 mice. Life Sci 63: 635-644.

9. Kano H, Mogami O, Uchida M (2002): Oral administration of milk fermented with Lactobacillus delbrueckii ssp. bulgaricus OLL1073R-1 to DBA/1 mice inhibits secretion of proinflammatory cytokines. Cytotechnology 40: 67-73.

10. So JS, Lee CG, Kwon HK, et al. (2008): Lactobacillus casei potentiates induction of oral tolerance in experimental arthritis. Mol Immunol 46: 172-180.

11. Amdekar S, Singh V, Singh R, et al. (2011): Lactobacillus casei reduces the inflammatory joint damage associated with collagen-induced arthritis (CIA) by reducing the pro-inflammatory cytokines: Lactobacillus casei: COX-2 inhibitor. J Clin Immunol 31: 147-154.

12. Amdekar S, Singh V, Kumar A, et al. (2014): Lactobacillus acidophilus protected organs in experimental arthritis by regulating the pro-inflammatory cytokines. Indian J Clin Biochem 29: 471-478.

13. Vyas U, Ranganathan N (2012): Probiotics, prebiotics, and synbiotics: gut and beyond. Gastroenterol Res Pract 2012: 872716.

14. Hakansson A, Molin G (2011): Gut microbiota and inflammation. Nutrients 3: 637-682.
15. Homayouni Rad A, Torab R, Ghalibaf M, et al. (2013): Might patients with immune-related diseases benefit from probiotics? Nutrition 29: 583-586.

16. Frei R, Akdis M, O’Mahony L (2015): Prebiotics, probiotics, synbiotics, and the immune system: experimental data and clinical evidence. Curr Opin Gastroenterol 31: 153-158.

17. Chung WS, Walker AW, Louis P, et al. (2016): Modulation of the human gut microbiota by dietary fibres occurs at the species level. BMC Biol 14: 3 .

18. Skivka LM (2015): Immunomodulatory properties of the human intestinal microbiota and prospects for the use of probiotics for prophylaxis and correction of inflammatory processes. Biotechnol Acta 8: 28-44.

19. Shokryazdan P, Faseleh Jahromi M, Navidshad B, Liang JB (2017): Effects of prebiotics on immune system and cytokine expression. Med Microbiol Immunol 206: 1-9.

20. Hansen CHF, Frokiaer H, Christensen AG, et al. (2013): Dietary xylooligosaccharide downregulates IFN-gamma and the low-grade inflammatory cytokine IL-1beta systemically in mice. J Nutr 143: 533-540.

21. Kim MH, Kang SG, Park JH, et al (2013): Short-chain fatty acids activate GPR41 and GPR43 on intestinal epithelial cells to promote inflammatory responses in mice. Gastroenterology 145: 396-406.e310.

22. Ang Z, Ding JL (2016): GPR41 and GPR43 in obesity and inflammation - protective or causative? Front Immunol 7: 28. 